\title{
A note on the rationing of divisible and indivisible goods in a general network
}

\author{
Shyam Chandramouli*and Jay Sethuraman ${ }^{\dagger}$
}

February 10, 2020

\begin{abstract}
The study of matching theory has gained importance recently with applications in Kidney Exchange, House Allocation, School Choice etc. The general theme of these problems is to allocate goods in a fair manner amongst participating agents. The agents generally have a unit supply/demand of a good that they want to exchange with other agents. On the other hand, Bochet et al. [7, 8] study a more general version of the problem where they allow for agents to have arbitrary number of divisible goods to be rationed to other agents in the network. In this current work, our main focus is on non-bipartite networks where agents have arbitrary units of a homogeneous indivisible good that they want to exchange with their neighbors. Our aim is to develop mechanisms that would identify a fair and strategyproof allocation for the agents in the network. Thus, we generalize the kidney exchange problem to that of a network with arbitrary capacity of available goods. Our main idea is that this problem and a couple of other related versions of non-bipartite fair allocation problem can be suitablly transformed to one of fair allocations on bipartite networks for which we know of well studied fair allocation mechanisms.
\end{abstract}

\footnotetext{
*IEOR Department, Columbia University, New York, NY; sc3102@columbia.edu

${ }^{\dagger}$ IEOR Department, Columbia University, New York, NY; jay@ieor.columbia.edu
} 


\section{Introduction}

The study of fair allocation on economic networks has gained a lot of importance in recent years with growing applications in many public policy domains like fair exchange of kidneys among patients [29], matching students to public schools [2], matching cadets in rotc [34] etc. The common theme in all these problems is that participating agents are in supply/demand of a unit indivisible good and the set of agents with whom they can share/recieve/supply this good is modeled by a link. These problems identify fair and strategyproof allocation mechanisms for agents in the network and thus is in very similar spirit of the classical marriage problem of Gale and Shapley [18].

On the other hand, Bochet et al. [7, 8] study the problem of fair division of a maximum flow in a capacitated bipartite network. This model generalizes and studies the exchange of divisible goods on a economic networks where agents have arbitrary supply/demand constraints. Note that, the aforementioned problems were typically unit supply/demand model. The common feature in both these research is that the associated market is moneyless, so that fairness is achieved by equalizing the allocation as much as possible. This last caveat is to account for additional considerations, such as Pareto efficiency and strategyproofness, that may be part of the planner's objective.

A well-studied special case of the Bochet et al. [7, 8] problem is that of allocating a single resource (or allocating the resource available at a single location) amongst a set of agents with varying (objectively verifiable) claims on it. This is the special case when there is a single supply node that is connected to every one of the demand nodes in the network by an arc of large-enough capacity. If the sum of the claims of the agents exceeds the amount of the resource available, the problem is a standard rationing problem (studied in the literature as "bankruptcy" problems or "claims" problems). There is an extensive literature devoted to such problems that has resulted in a thorough understanding of many natural methods including the proportional method, the uniform gains method, and the uniform losses method. A different view of this special case is that of allocating a single resource amongst agents with single-peaked preferences over their net consumption. Under this view, studied by Sprumont [35, Thomson [39] and many others, the goal is to design a mechanism for allocating the resource that satisfies appealing efficiency and equity properties, while also eliciting the preferences of the agents truthfully. The uniform rule, which is essentially an adaptation of the uniform gains method applied to the reported peaks of the agents, occupies a central position in this literature: it is strategy-proof (in fact, group strategy-proof), and finds an envy-free allocation that Lorenz dominates every other efficient allocation; furthermore, this rule is also consistent. A natural two-sided version of Sprumont's model has agents initially endowed with some amount of the resource, so that agents now fall into two categories: someone endowed with less than her peak is a potential demander, whereas someone endowed with more than her peak is a potential supplier. The simultaneous presence of demanders and suppliers creates an opportunity to trade, and the obvious adaptation of the uniform rule gives their peak consumption to agents on the short side of the market, while those on 
the long side are uniformly rationed (see [23, 6]). This is again equivalent to a standard rationing problem because the nodes on the short side of the market can be collapsed to a single node. The model we consider generalizes this by assuming that the resource can only be transferred between certain pairs of agents. Such constraints are typically logistical (which supplier can reach which demander in an emergency situation, which worker can handle which job request), but could be subjective as well (as when a hospital chooses to refuse a new patient by declaring red status). This complicates the analysis of efficient (Pareto optimal) allocations, because short demand and short supply typically coexist in the same market.

As mentioned earlier, Bochet et al. [7, 8]. work with a bipartite network in both papers and assume that each node is populated by an agent with single-peaked preferences over his consumption of the resource: thus, each supply node has an "ideal" supply (its peak) quantity, and each demand node has an ideal demand. These preferences are assumed to be private information, and Bochet et al. [7, 8] propose a clearinghouse mechanism that collects from each agent only their "peaks" and picks Pareto-optimal transfers with respect to the reported peaks. Further, they show that their mechanism is strategy-proof in the sense that it is a dominant strategy for each agent to report their peaks truthfully. While the models in the two papers are very similar, there is also a critical difference: in [8], the authors require that no agent be allowed to send or receive any more than their peaks, whereas in [7] the authors assume that the demands must be satisfied exactly (and so some supply nodes will have to send more than their peak amounts). The mechanism of Bochet et al. - the egalitarian mechanism-generalizes the uniform rule, and finds an allocation that Lorenz dominates all Pareto efficient allocations. Later, Chandramouli and Sethuraman [12, 11] show that the egalitarian mechanism is in fact strongly invariant, peak and link group strategyproof: it is a dominant strategy for any group of agents (suppliers or demanders) to report their peaks truthfully. Szwagrzak [36] studies the property of contraction invariance of an allocation rule: when the set of feasible allocations contracts such that the optimal allocation is still in this smaller set, then the allocation rule should continue to select the same allocation. He shows that the egalitarian rule is contraction invariant. These results suggest that the egalitarian mechanism may be the correct generalization of the uniform rule to the network setting.

Our research is motivated by these results. From the results above, the generalized model of allocation of divisible goods on bipartite networks is well understood. On the contrary, there has not been a much focus in the litreature on fair mechanisms on non-bipartite networks. Our contribution can be viewed in some sense a generalization of identifying a fair maximum matching on general networks to that of identifying a fair maximum b-matching problem on non-bipartite networks. Our contributions parallel the contribution of Bochet et al. [7, 8] in generalizing unit capacity models.

Specifically, we are given a non-bipartite network $G=(N, E)$, and we think of $N$ as the set of agents involved in this network. Each arc $(i, j) \in E$ connects two participating agents and has capacity $u_{i j} \geq 0$. There is a single commodity (the resource) that is available at each node and 
needs to be exchanged with the neighbors on the network: we assume that node $i$ has $b_{i}$ units of the resource. The capacity of an arc $(i, j)$ is interpreted as an upper bound on the direct transfer from supply node $i$ to demand node $j$. The agents derive utility whenever they exchange a good with their neighbors. The goal is to find a maximum "fair" exchange among participating agents , while also respecting the capacity constraints on the arcs. We would describe in more detail our model in the later sections. We describe below the connections of this model with litreature.

- Connection to Kidney Exchange Problem: The kidney exchange problem of [29] and the subsequent heaavy litereature, study a unit exchange problem between patients who are connected to their compatible donors through links in the network. Each agent needs exactly one unit of good (in this case, kidney). Since, not every patient is compatible with everyone else, we are naturally poised with the problem of not able to match every patient with his compatible donor, hence the goal of the study is to identify maximum number of matches as possible. In a combinatorics view, this problem can be viewed as one of finding a maximum matching on a non-bipartite network. When the set of maximum matchings is not unique, the mechanism designer is forced to pick one such maximum matching. From an economics perspective, the mechanism designer would like to satisfy certain fairness objectives like Lorenz Dominance, Pareto Optimality etc. and also incentive compatible conditions. Roth et al. 29] construct the "Egalitarian Mechanism" - which is a randomized lottery mechanism over the set of maximum matchings. They identify a fair and strategyproof matching. As described above, our problem is one in which each agent $i$, in the networks has arbitrary units $b_{i}$ and derives utility with every exchange of that good with his/her neighbors in the network. Since the goods are indivisible, our model generalizes the kidney exchange problem to multiple demands and from a combinatorics perspective, generalizes to one one of identifying a maximum b-matching on a non-bipartite network.

- Ordinal transportation problem: Ordinal transportation problem of Balinski and Yu [4], study the problem of identifying a stable b-matching on bipartite networks. We differ from their study in many ways. Our model is more general non-bipartite networks, our preference structure is not ordinal (agents are indifferent from whom they recieve the goods from, the utility structure is single peaked) and we are more interested in fairness notions like pareto optimality and envyfreeness versus the notion of stability studied by Balinski.

Our Contributions; Our main contribution in this paper is a generalized egalitarian mechanism that is Lorenz dominant, pareto optimal, envyfree and strategyproof for agents participating in a non-bipartite network. We identify this in both the divisible and indivisible goods case. We do so by transforming each of these non-bipartite networks to a suitable bipartite network. We solve the fractional matching problem on this bipartite network and show how to construct a lottery over integral b-matchings in the original network. 
The rest of the paper is organized as follows: in Section 2 we consider the divisble goods case and in Section 3 we consider the indivisble goods case. Finally in Section ??, we study extensions to problems with capacities, weighted matchings and general s-t networks, non-pairwise exchange

\section{Definitions:}

Here we describe some of the common notions that are used in this paper. We are describing it explicitly here because it is the same terminology that we use across our models.

Pareto Optimality: A feasible net transfer $x$ as defined in the previous section is Pareto Optimal if there is no other allocation $x^{\prime}$ such that every agent is weakly better off and atleast one agent is strictly better off in it. In mathematical terms, if $R_{i}$ and $I_{i}$ denote the preference and indifference relations respectively for agent $i$, then

$$
\left\{\forall i: x_{i}^{\prime} R_{i} x_{i}\right\} \Longrightarrow\left\{\forall i: x_{i}^{\prime} I_{i} x_{i}\right\}
$$

Lorenz Dominance: A solution is Lorenz Dominant inside the pareto optimal set if for any $z \in \mathbb{R}^{V}$, write $z^{*}$ for the order statistics of $z$, obtained by rearranging the coordinates of $z$ in increasing order. For $z, w \in \mathbb{R}^{V}$, we say that $z$ Lorenz dominates $w$, written $z L D w$, if for all $k, 1 \leq k \leq n$

$$
\sum_{a=1}^{k} z^{* a} \geq \sum_{a=1}^{k} w^{* a}
$$

Lorenz dominance is a partial ordering, so not every set, even convex and compact, admits a Lorenz dominant element. On the other hand, in a convex set $A$ there can be at most one Lorenz dominant element. The appeal of a Lorenz dominant element in $A$ is that it maximizes over $A$ any symmetric and concave collective utility function.

No Envy: A rule $x \in \mathcal{F}(G, b, u)$ satisfies $N o$ Envy if for any preference profile $R \in \mathcal{R}^{S \cup D}$ and $i, j \in S$ such that $x_{j} P_{i} x_{i}$, there exists no $x^{\prime}$ such that

$$
x_{k}=x_{k}^{\prime} \text { for all } k \in S \backslash\{i, j\} \text {; for all } l \in D \text { and } x_{i}^{\prime} P_{i} x_{i}
$$

Strategyproof: A rule $x$ on $(G, b, u)$ is strategyproof if for all $R \in R^{S \cup D}, i \in V$ and $R_{i}^{\prime} \in \mathcal{R}$

$$
x_{i}(R) R_{i} x_{i}\left(R_{i}^{\prime}, R_{-i}\right)
$$

\section{Model 1: With preference indifference, divisible goods}

In this section we consider the version of the problem where the nodes of the network are populated by agents. Each node has a specific number of units of a particular good that they can exchange 
with the agents that they are connected with. Thus, our problem becomes one of exchanging a single commodity among the set of agents $V$ using the set $E$ of edges.

An exchange of the commodity among the agents is realized by a b-matching $f$, which specifies

the amount of the commodity exchanged among the agents $i$ and $j$ using the edge $(i, j) \in E$. The flow $f$ induces an allocation vector for each agent as follows:

$$
\text { for all } i \in V: x_{i}(f)=\sum_{j \in N(i)} f_{i j}
$$

As we shall see in a moment, agents only care about their net transfers, and not on how these transfers are distributed across the agents on the other side.

In the following sections, we assume that the peaks of the agents are fixed, and focus our attention on mechanisms that elicit preferences from the agents on their connectivity and maps the reported connectivity for each preference profile to a unique b-matching.

To summarize: agents arrive to the market with a specified number of divisible goods and report the agents with whom they can have exchanges; They are preference homogeneous in the sense that they are indifferent between whom they exchange. the allocation rule is applied to the graph $G$ with edge-capacities $u$, and the data $b$. Our focus will be on mechanisms in which no agent has an incentive to misreport his compatible neighbors and also efficient.

\section{Allocation Rules}

We define an allocation on the edges as $f_{a b}, a b \in E$ as feasible, if $f_{a b}=f_{b a} \forall a b \in E$ and the flow induced on the nodes by $f$ (as $x$ defined earlier) and for feasibility, $x_{a} \leq b_{a} \forall a \in V$. Any rule which picks an allocation from this set is called a feasible allocation rule. In the rest of the section, we discuss the egalitarian rule for this model.

Given a network $(G, N, E)$, we make the following transformation to construct the bipartite network $\left(G_{b}, V_{b}, E_{b}\right)$. We represent $V_{b}=A \cup B$ where $A=V$ and $B=V$. are either sides of the network. There is an edge between agent $i \in A$ and $j \in B$ only if there is an edge $i j$ in the given network $G$. Connect the agents in $A$ to a supply node $s$ and the agents in $B$ to a sink node $t$. We refer to as a flow, any feasible shipment of goods from the source node to the sink node.

Lemma 1 Every feasible flow in the modified network $G_{b}$ corresponds to a feasible allocation (exchange) in the original network $G$. BIMS's Egalitarian rule on the modified network $G_{b}$ results in a pareto optimal, lorenz dominance, envy free and peak strategy proof allocation for the agents in the original network $G$.

Proof : Consider a solution $\left(y_{a}, y_{b}, g_{a b}\right)$ for the agents in the modified bipartite network where $y_{a}, y_{b}$ refers to an allocation for agent $a \in A$ and $b \in B$ respectively. $g_{a b}$ is the flow on the edge $a b$. We will try to show that every feasible solution in the modified bipartite network maps to a solution in the original network and vice-versa. Once we have that, the second part of the 

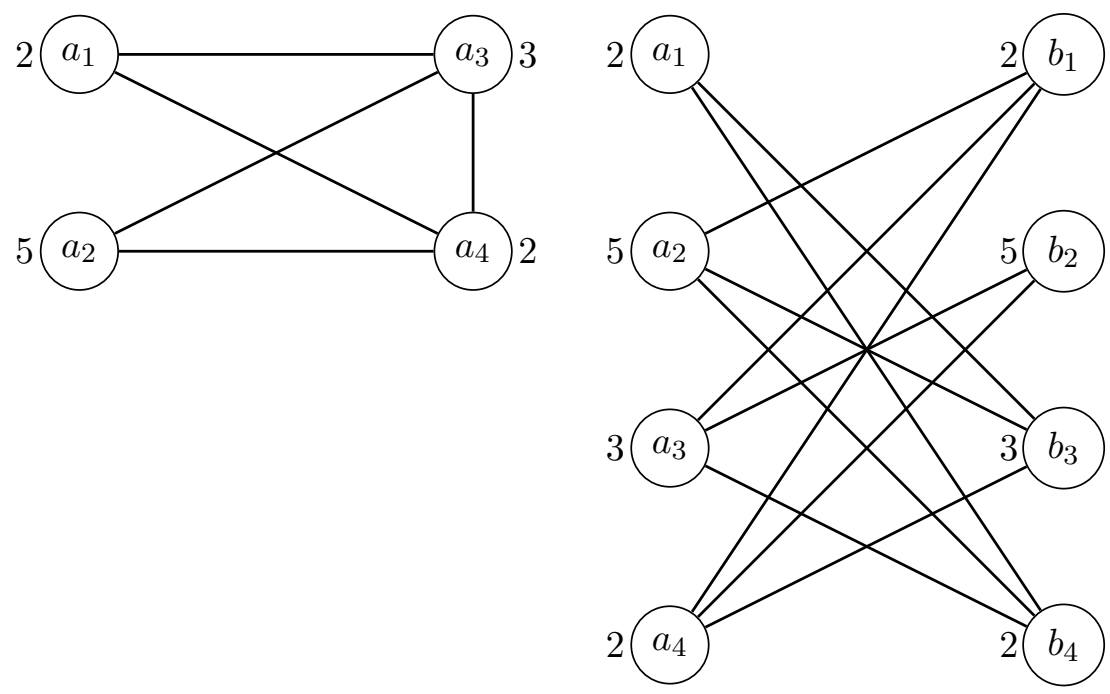

Figure 1: Transformation to bipartite network

statement follows (As Bochet et al. [7, 8] have established the fairness properties of egalitarian mechanism in bipartite networks).

Firstly, consider a feasible exchange in the original problem. Denote the solution by $\left(x_{a}, f_{a b}\right)$ for agent $a, b$ in the network. $x, f$ are node and edge allocations respectively. In the modified network, set $g_{a_{i} b_{j}}=g_{a_{j} b_{i}}=f_{a_{i} a_{j}} \forall i, j \in V$. Clearly, this solution is feasible in the modified network and $y_{a_{i}}=y_{b_{i}}=x_{a_{i}} \forall i \in V$.

Now, consider a solution $\left(y_{a}, y_{b}, g_{a b}\right)$ in the modified bipartite network. Construct the following solution, $f_{a_{i} a_{j}}=\left(g_{a_{j} b_{i}}+g_{a_{i} b_{j}}\right) / 2 \forall i, j \in V$. This also defines a node allocation in the original network with $x_{a_{i}}=\left(y_{a_{i}}+y_{b_{i}}\right) / 2 \forall i \in V$. This is a feasible allocation since, $f_{i j}=f_{j i} \forall i j \in E$ and $x_{i} \leq b_{i} \quad \forall i \in V$.

Hence, the case of exchanging divisible goods in an economic network is done by a simple transformation into a bipartite network. The mechanism is also peak strategyproof if the preference profiles are private information of the agents. In the next section, we discuss the case of exchanging indivisible goods among agents in a network. We show that there is no mechanism that is peak strategyproof but we design a mechanism which is egalitarian in nature and retains many other attractive properties. 


\section{Model 2: With preference indifference, Indivisible goods}

In this section, we focus on allocation rules, which allows only integer allocations to the agents in the network. To summarize: agents arrive to the market with a specified number of indivisible goods and report the agents with whom they can have exchanges; They are preference homogeneous in the sense that they are indifferent between whom they exchange. the allocation rule is applied to the graph $G$ with edge-capacities $u$, and the data $b$. Our focus will be on mechanisms in which no agent has an incentive to misreport his connectivity.

At this juncture, we would like to highlight an important difference with that of the bipartite network structure here. The egalitarian rule of Bochet et al. [7, 8] constructs a fair allocation for agents in a bipartite network with divisible goods. This rule is a generalization of the well known Sprumont's [35] uniform rule. On the other hand, when we are allocating a set of indivisible goods among agents, Klaus et al. [17] proposed the probablistic uniform rule in the single agent model. Their main contribution is the fact that there is no net utility loss for agents in both divisible/indivisible models. The fractional part of an agents allocation (expected utility from this mechanism) is the probability with which he has a claim on an extra unit of good.

Klaus et al. [17] mechanism is based on a simple idea that at each bottleneck point of the Sprumont's model, we randomize over all possible feasible allocations. Similarly, we define an egalitarian mechanism for indivisible goods. The idea is to randomize over all possible feasible flows at each bottleneck. The utility for the agents in the divisible goods case is same as the expeced utility in the indivisible goods case.

This is not the case in non-bipartite networks. The net utility (sum total of utilities for all agents) is not the same in divisible/indivisible goods case. For a simple example, consider a traingular network with nodes $(a, b, c)$ each with peak $=1$; In the divisible goods case, a pareto allocation would assign 1 unit to each node ( 0.5 units on each edge). The net social utility is 3units in this case. If the goods are indivisible, only 1 of the 3 edges can be picked in any maximal allocation (pareto). The net social utility is 2 units. In a fair allocation, we would pick each edge with equal probability, giving an allocation of $2 / 3$ for each agent. Hence, there is a clear gap in the achievable utility between divisible and indivisible goods case in non-bipartite networks.

Hence, in this section we aim to identify an egalitarian mechanism for this model where the agents arrive with indivisible goods. We reduce it to a suitable bipartite network and apply the well-known fair allocation rules on this modified network.

Extensions of bipartite allocation rules: We note another property of the mechanisms which would be different when we move to non-bipartite networks. "Extension" is the property that when we restrict ourselves to bipartite networks, the mechanisms that we construct should boil down to the familiar fair allocation rules. Due to the "utility gap" we discussed earlier, the divisible

and indivisible rules on non-bipartite networks would reduce to their respective counterparts on bipartite networks. Though on bipartite networks the rules look similar, they need to be adjusted 
for the "utility gap" when we move to non-bipartite networks. We would discuss this aspect in further detail in later sections

\section{b-matchings}

b-matchings is a generalized notion of a matching type problems on networks. Given a network $G$ and a positive number $b_{i}$ for each node $i \in V$ and capacity $u_{e}$ for each edge $e \in E$ We define the $u$-capacitated $b$-matching or $(\mathrm{b}, \mathrm{u})$ matching is a vector $x$ such that

$$
\begin{gathered}
x_{i}(f) \leq b_{i} \text { for all } i \in V \\
0 \leq f_{i j} \leq u_{i j} \text { for all } i j \in E
\end{gathered}
$$

In the case when all the $u_{e}=\infty$ and $b_{i}=1$, we arrive at the problem of finding a matching in network $G$. The set of all $x$ which satisfies [5] is the set of all feasible b-matchings. Clearly, a given graph $G$ can have more than one b-matching. From an operations research as well as economics point of view we will be interested in finding the maximum b-matching among all feasible b-matchings. Here, the maximum b-matching is the one with the highest $\sum_{i} x_{i}$ among all the feasible b-matchings.

A maximum weight $\mathrm{u}$-capacitated b-matching problem can be solved in strongly polynomial time by a reduction to the maximum weight b-matching problem. Following is a linear programming formulation of the maximum weight b-matching problem:

$$
\begin{gathered}
\operatorname{Max} \sum_{i} \sum_{j} X_{i j} \\
\text { subject to } \\
\sum_{j \in N(i)} X_{i j} \leq b_{i} \quad \forall i \in V(G) \\
X_{i j} \in Z
\end{gathered}
$$

The maximum weight u-capacitated b-matching problem would encode the additional constraint $X_{i j} \leq u_{i j} \forall i j \in E(G)$. In this section, we assume $u_{i j}=\infty$ but we discuss the capacitated case in later sections.

Algorithms for finding a maximum weight b-matching: Cook and Cunningham [15] discuss polynomial algorithms to solve the b-matching problem. We briefly discuss about the b-matching matroid. This discussion follows from the well-known matching matroid formulation. For details on matching matroid, refer to cook [15].

For an undirected graph $G(V, E)$ define, $\mathcal{I}=\left\{x_{v e c} \mid x_{v e c}\right.$ is the node allocation from some b-matching of $\left.\mathrm{G}\right\}$ where $x_{v e c} \in R^{V}$, the $i^{\text {th }}$ component of the vector, $x_{v e c}^{i}=x_{i}$ where $x_{i}$ is the number of times node $i$ is matched in that particular b-matching. Then the pair, $(V, \mathcal{I}):=M$ is a matroid. We will refer to it as the b-matching matroid. A base in $M$ is a vector $x_{v e c}$ that is generated by some maximum b-matching of $G$. 
The "rank" function : $2^{V} \rightarrow Z^{+} \cup\{0\}$ of the matroid $M$ is defined to be $\operatorname{rank}(\mathrm{S})=$ $\max _{I \subset S, I \in \mathcal{I}}|I| \forall S \subseteq V$. The rank function is submodular and in our context $\operatorname{rank}(\mathrm{S})$ can be interpreted as the size of a maximum b-matching in the set S. $P_{\text {rank }}$ refers to the polymatroid associated with this particular rank function and is given by

$$
P_{\text {rank }}=\left\{x \in R^{V} \mid x \geq 0, x(S) \leq f(S), \quad \forall S \subseteq V\right\}
$$

where $x(S)=\sum_{i \in S} x_{i}$

From an Operations Research perspective, we have obtained an optimal solution to the maximum weight b-matching problem. But the aforementioned linear program can have many solutions. From an Economics perspective, we would like to pick a particular maximum weight b-matching from the optimal set such that the utility of the agents satisfy fairness constraints. Inoder to find such an allocation, we would understand the structure of maximum weight bmatchings for the uncapacitated problem in the rest of this section. Towards the end, we analyze the case with capacities.

\section{Pareto Optimality}

A feasible allocation $x \in \mathcal{A}(G)$ is Pareto Optimal if for any other $x^{\prime} \in \mathcal{A}(G)$ we have

$$
\left\{\text { for all i: } x_{i}^{\prime} R_{i} x_{i}\right\} \Longrightarrow\left\{\text { for all i: } x_{i}^{\prime} I_{i} x_{i}\right\}
$$

i.e. there is no other allocation $x^{\prime}$ that is weakly better for every agent and strictly better for at least one agent in the allocation $x$.

Lemma 2 The set of pareto optimal allocations is equivalent to the set of maximum weight $b$ matchings

Proof: If $x$ denotes the utiliy profile of the agents in the network, then the size of the bmatching that produced this utility profile is $x / 2$. As each exchange between 2 agents on a network adds an utility of one to each of those agents.

Suppose, we have an exchange of goods in the network that is produced by a maximum bmatching resulting in the allocation profile $x$. Since, it is a maximum b-matching, we cannot have more feasible exchanges in this network. There is no alternating path of odd length connecting two unsaturated nodes. Any other path of even length from an unsaturated node $a$, to another unsaturated node $b$ will result in another maximum b-matching with the utility of node $b$ strictly below the current solution. Hence, picking any maximum b-matching to the problem should result in an pareto optimal allocation for the agents.

Now, suppose $x$ is a pareto optimal allocation for the agents in the network, then there exists no allocation $y$ in the network such that every agent is weakly better under allocation $y$, and there is atleast one agent strictly better off under $y$. This implies, there is no alternating path 
of odd length connecting two unsaturated nodes. This implies the given solution is a maximum b-matching.

The following generalization of Gallai and Edmonds theorem further helps us understand the structure of pareto optimal solutions

\section{Gallai - Edmonds Decomposition}

Gallai and Edmonds decompose any given network into smaller sub-components namely, over demanded, under demanded and pefectly demanded components. Bochet et al. [7, 8] use this decmposition to establish the structure of pareto optimal allocations in bipartite networks. Roth et al. [29] establish the structure of Pareto-efficient pairwise matchings using the Gallai-Edmonds decomposition (GED). We generalize the GED idea to our problem where the nodes have arbitrary peaks associated with them. Hence, we obtain a characterization of pareto efficient b-matchings in this problem. $M$ is the set of maximum b-matchings and let $\mu$ denote an arbitrary matching in the set $M, \mu(i)$ denotes the matched neighbors of agent $i$ in matching $\mu$. Setting up the notation partition $V$ as $\left\{V^{U}, V^{O}, V^{P}\right\}$ such that

$$
\begin{aligned}
V^{U} & =\{i \in V: \exists \mu \in M \text { s.t. } i \in \mu(i)\} \\
V^{O} & =\left\{i \in V \backslash V^{U}: \exists \text { set of agents } \tilde{j} \in V^{U} \text { s.t. } \sum_{j \in \tilde{j}} n_{i, j}=b_{i}\right\}, \\
V^{P} & =V \backslash\left(V^{U} \cup V^{O}\right)
\end{aligned}
$$

where $n_{i, j}=1$ if $i, j$ is matched in a feasible solution. $V^{U}$ is the set of agents unmatched $\left(x_{i}<b_{i}\right)$ in atleast one maximum b-matching. $V^{O}$ is the set of agents completely matched $\left(x_{i}=b_{i}\right)$ in every maximum b-matching and have atleast 1 neighbor in $V^{U} . V^{P}$ is the set of agents who are again perfectly matched but does not have a link with any agent in $V^{U}$.

Let $I \subseteq V$ and $N(I)=\{j \mid i j \in E, i \in I\}$. Then $(I, N(I))$ is a reduced sub problem of the original problem.

Lemma 3 Let $I=V \backslash V^{0}$ and let $\mu$ be a pareto-efficient matching for the original problem $(\mu(i)$ is the set of neighbors that is matched to an agent $i$ ), then

- For any agent $i \in V^{0}, \mu(i) \subseteq V^{U}$

- For any even comoponent $(J, N(J))$ such that $J \subseteq V^{P}$ and for any agent $i \in J, \mu(i) \in J \backslash i$

- For any odd comoponent $J(\|J\|>=2)$, the maximum size of b-matching within every odd comoponent is $\Sigma_{j=1}^{|J|} b_{j}-1$. Moreover, for any odd comoponent $\left(J, R_{J}\right)$, either

- one and only one agent $i \in J$ is matched with a agent in $V^{O}$ under the pareto efficient matching $\mu$ whereas all remaining agents in $J$ are matched within so that $\mu(j) \in J \backslash\{i, j\}$ for any agent $j \in J \backslash\{i\}$ or 


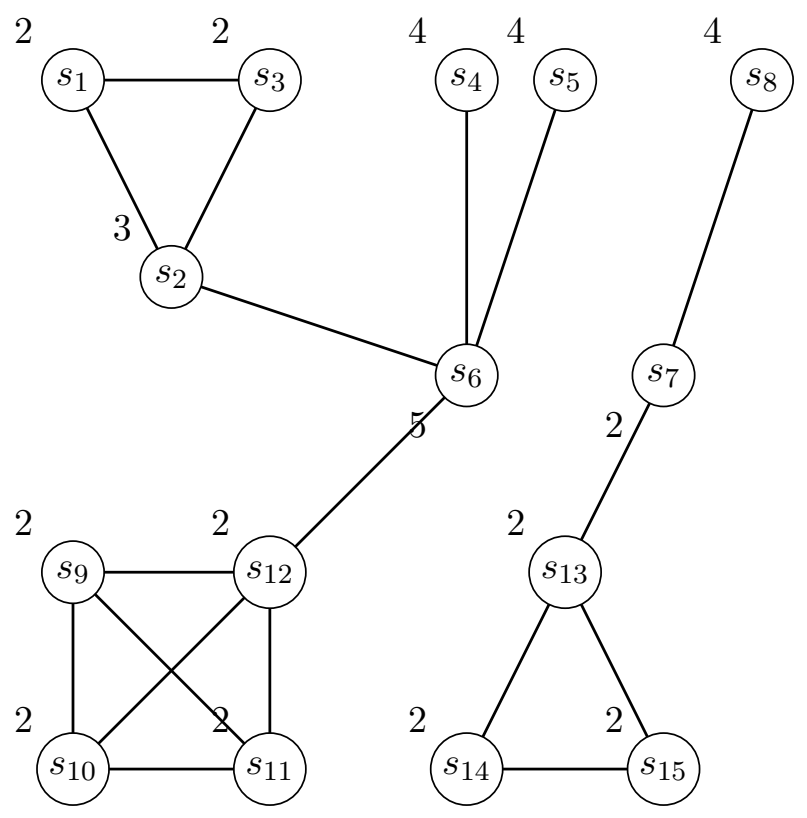

Figure 2: GED of a non-bipartite network with arbitrary peaks; In this figure, $\left\{s_{6}, s_{7}\right\} \in$ $V^{O},\left\{s_{1}, s_{2}, s_{3}, s_{4}, s_{5}, s_{8}\right\} \in V^{U},\left\{s_{9}, s_{10}, s_{11}, s_{12}, s_{13}, s_{14}, s_{15}\right\} \in V^{P}$

- one agent $i \in J$ remains unsaturated under the Pareto-efficient matching $\mu$ whereas all remaining agents in $J$ are saturated so that $m u(j) \in J \backslash\{i, j\}$ for any agent $j \in J \backslash i$

Proof : The Gallai-Edmonds decomposition precisely proves the above lemma when all the peaks $b_{j}=1$. Roth et al. [29] which this paper builds upon has a statement when the nodes have unit capacity. Now, lets construct a matching instance of the given b-matching problem. From the given graph $(G, V, E)$ construct the following graph $\left(G^{\prime}, V^{\prime}, E^{\prime}\right)$ : Create $b_{i}$ duplicate copies of node $i$ with unit peak each. Label these nodes as $\left(i^{1}, i^{2} \ldots i^{b_{i}}\right)$. In the graph $G^{\prime}$, nodes $i^{k},\left(k \in 1,2, . . b_{i}\right)$ and $j^{l},\left(l \in 1,2, . . b_{j}\right)$ is connected by an edge if $i j$ is conencted in the original graph $G$. There is no edge between $i^{k}$ and $i^{r},\left(k, r \in\left(1,2, \ldots b_{i}\right)\right)$ in $G^{\prime}$. Now, we have a graph with unit peaks. Applying the GED on this unit graph would establish the result.

We just stress a bit on the third statement. When $(|J|=1)$ the odd component has only 1 element, then there is no b-matching within that component. When $|J|>=2$, we highlight that the maximum size of any matching within every odd component in a unit capacity graph is one less than the number of nodes in the component (refer to Roth et al. [29] for a proof). By symmetry, all the duplicate (identical) copies of a node will be in the same component. Hence, we can shrink these duplicate nodes into the parent node with original peak. Thus, from the decomposition above, the sum of the peaks of the agents in a odd component is $\Sigma_{j=1}^{|J|} b_{j}$ and the result follows. 


\section{$\underline{\text { Allocation Rules }}$}

\section{Utility Profile:}

A utility profile $U \in R^{V}$ is said to be a feasible utility profile if the profile is an outcome of a feasible b-matching in the network. Since, we are looking for pareto optimal solutions, the outcome of our mechanisms is an utility profile induced by a maximum b-matching in the network.

A mechanism is deterministic if for a given network, it picks a maximum b-matching as an outcome from the set of maximum b-matchings. Roth et al. [29] study priority mechanisms in the context of deterministic mechanisms. They also show establish that the randomized "Egalitarian" mechanism is superior in the sense that it not only retains the efficiency, strategyproof properties of deterministic mechanism, but it also produces an outcome which is envy free and strongly efficient in the sense of lorenz dominance. The mechanism of Roth et al. is a "lottery" mechanism, that randomly picks a maximum b-matching from the pareto optimal set. The distribution or lottery is chosen in such a way that the aforementioned economic properties are exhibited by the mechanism. We define this more mathematically below.

\section{Lottery Mechanism:}

Let $\mathcal{U}$ be the set of all b-matchings in $G$. A matching lottery $l: P_{\mu}, \mu \in M$ is a probability distribution over $\mathcal{U}$. For each matching $\mu \in \mathcal{U}, P_{\mu}, \mu \in[0,1]$ is the probability of choosing matching $\mu$ in lottery $\mathrm{l}$, and $\sum_{\mu \in \mathcal{U}} P_{\mu}=1$. A matching lottery $l$ can be also viewed as a fractional b-matching which

is defined to be a convex combination of several integral b-matchings. Let $\mathcal{L}$ be the set of matching lotteries. Given $l \in \mathcal{L}$, define the utility $x_{i}^{l}$ of vertex i to be the expected total exchanges that $\mathrm{i}$ is involved, i.e., $x_{i}^{l}=\sum_{\mu \in \mathcal{U}} l_{\mu} x_{i}^{\mu}$. Define the utility profile induced by lottery $l$ to be the vector $x_{l}=\left(x_{i}^{l}\right), i \in V$. Let $P=x_{l}, l \in \mathcal{L}$ be the set of all feasible utility profiles.

\section{Egalitarian Mechanism: Description and Properties}

It is now clear from our definition that a feasible utility profile can be understood as a fractional b-matching (a convex combination of integral b-matchings). The $P_{\text {rank }}$ polymatroid we defined earlier is exactly the set of feasible utility profiles.

Roth et al. 29] described the Egalitarian Mechanism for the pairwise kidney exchange problem. Jianli et al. [1] develop a water filling algorithm which gives a simpler and intuitive proof of the Roth's mechanism. Here, we use the ideas from jianli et al. to develop an Egalitarian Mechanism for the generalized maximum indivisible exchange problem. The idea is similar in the sense that the problem on a general network can be suitably reduced to a problem on a bipartite network. This can be done if one could establish the equivalence between the solutions in both network. Once the above step is completed, we could run the familiar allocation rules for bipartite networks to establish a allocation for our original general network. We describe this procedure 
below in more detail

Step 1: Transformation to equivalent bipartite network: We construct the following two sided bipartite flow network. The left side of the bipartite network consists of nodes for each agent $i$.

Construction of Nodes: Define $\mathcal{C}=\left\{C_{1}, C_{2} \ldots . C_{k}\right\}$ as the set of odd components in the underdemanded component $V^{U}$ where $k=|C|$. Construct the following bipartite graph $G_{B}=(A, B, E)$ where each node in $A$ corresponds to a node in $V$. We use $A^{C_{1}}, \ldots, A^{C_{k}}$ to denote $k$ disjoint sets corresponding to $C_{1}, C_{2}, . . C_{k}$ respectively. Let $A^{P O}=\left\{a_{i} \mid i \in V^{P} \cup N^{O}\right\} ; A^{U}=\left\{a_{i} \mid i \in V^{U}\right\}$.

The construction of $\mathrm{B}$ is as follows. B Can be partitioned into 3 parts: $B^{P O} \cup B^{O} \cup B^{C}$. Each node in $B^{P O}$ corresponds to a node in $B^{P} \cup B^{O}$. We use $c_{i} \in B^{P O}$ to denote the node corresponding to $i \in B^{P} \cup B^{O}$. Each node in $B^{P}$ corresponds to a node $V^{P}$. We use $c_{i}^{\prime} \in B^{O}$ to denote the node corresponding to $i \in V^{P}$. $B^{C}$ consists of $k$ disjoint sets $B^{C_{1}}, B^{C_{2}} \ldots . B^{C_{k}}$, where $B^{C_{i}}$ contains a node with value $\Sigma_{j \in C_{i}} b_{j}-1$ (this is the maximum b-matching within a particular odd component) if and only if $\left|B^{C_{i}}\right| \geq 2 ; B^{C_{i}}$ is empty otherwise.

Construction of Edges:

(1) For each $i \in V^{P} \cup V^{O}$, we have edge $\left(a_{i}, c_{i}\right) \in E$ where $a_{i} \in A^{P O}$ and $c_{i} \in B^{P O}$

(2) For a vertex $a_{i} \in A^{C}$ and another vertex $c_{j}^{\prime} \in B^{O}$ we have $\left(a_{i}, c_{j}^{\prime}\right) \in E$ if $(i, j) \in E$

(3) For each vertex, $a \in A^{C_{i}}$ add an edge to the node in $B^{C_{i}}$.

Step 2: Apply the egalitarian mechanism (BIMS): Apply the Egalitarian Mechanism for indivisible goods in a bipartite network.

Step 3: Construct a randomized mechanism: lottery over b-matchings: The egalitarian mechanism outcomes a fractional utility profile for the agents. From theorem 11 later, it is clear that such a utility profile is actually feasible and can be generated as a lottery over integral maximum b-matchings. Every time, we generate a maximum b-matching with this probability profile and conduct exchanges on the network.

Example: Consider the original network as given in figure 2, the bipartite transformation outlined in step 1 is shown in figure 3. When the egalitarian mechanism is applied to this bipartite network, we get the following utility profile for the agents: Every agent $i \in V^{P} \cup V^{O}$ receive their allocation $=$ peak. The agents $s_{1}, s_{2}, s_{3}$ receive 2 units of utility each. The utility of agents $s_{2}, s_{4}, s_{5}$ is $\frac{7}{3}$ each. This fractional utility is obtained as a lottery over integral b-matchings. In this case, 2 units of agents $s_{4}, s_{5}$ is always exchanged with agent $s_{6}$. $s_{2}$ has to exchange 2 units within its odd component in every maximum b-matching. Agents $s_{2}, s_{4}, s_{5}$ compete for the extra unit of exchange that $s_{6}$ can do. The egalitarian mechanism picks any of the possibility with a probability of $\frac{1}{3}$. 


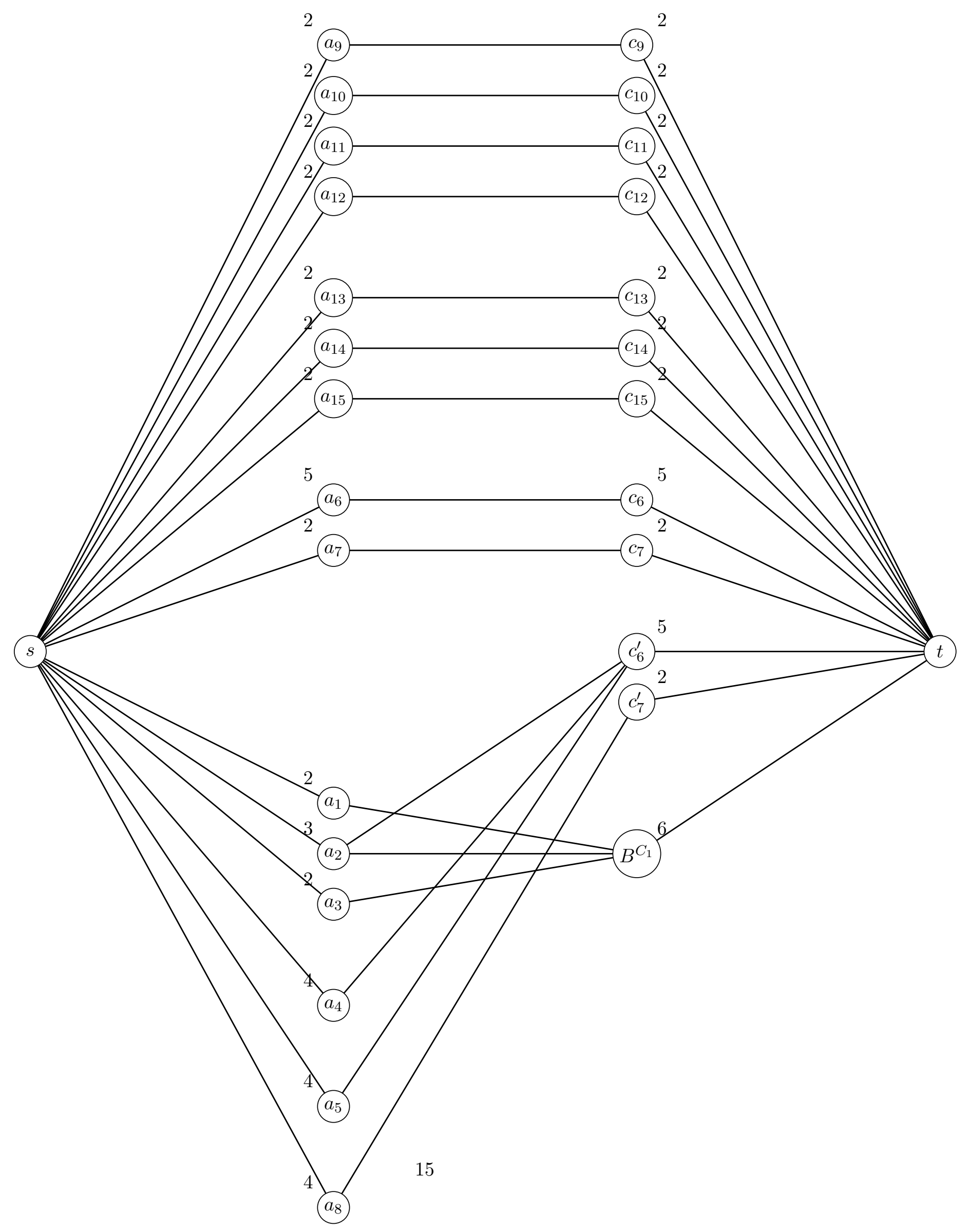




\section{Egalitarian Mechanism: Linear Programming Approach}

1) Step 1. Solve the linear program $L P_{1}$

$$
\begin{gathered}
\text { Maximize } \lambda_{1} \\
\text { subject to } \\
x_{v}=\lambda_{1}, \forall v \in V, x \in P_{\text {rank }}
\end{gathered}
$$

We call an element $v \in V$ a tight element if $x_{v}$ participates in some tight constraint in $P_{\text {rank }}$. Let $D_{1}$ be the set of tight elements. In other words, increasing $x_{v}$ would violate some constraint in $P_{\text {rank }}$ when other $x_{u}$ for $u \neq v$ are fixed. In many linear programming algorithms, we can easily detect such tight elements. Another way to test the tightness of an element is to solve a closely related linear program in which we fix other $x_{u}$ and maximize $x_{v}$.

(2) In general, at Step $k$, we solve the linear program

$$
\begin{gathered}
L P_{k}=\text { maximize } \lambda_{k}, \text { subject to } x_{v}=\lambda_{j}, \quad \forall v \in D_{j} \forall j<k, \\
x_{v}=\lambda_{k}, \forall v \in V \backslash \cup_{j<k} D_{j}, \quad x \in P_{\text {rank }} .
\end{gathered}
$$

Let $D_{k}$ be the set of elements that become tight in this step.

(3) The algorithm return $x=x_{v}=\lambda_{j}$ for $v \in D_{j}$ if $\cup_{j=1}^{k} D_{j}=V$

Lemma 4 Every feasible maximum flow on the modified bipartite network is equivalent to a feaible utility profile in the original network

Proof: A utility profile in the original network is induced by a maximum b-matching $\mu$ or a lottery (linear combination) over the set of maximum b-matchings. Also, it is well known that the polytope of maximum flows is convex with integral extreme points. Hence, to prove that every utility profile in original network implies a feasible maximum flow in the modified network, it is sufficient to prove the statement only for integral utility profiles (as every deterministic maximum b-matching corresponds to a integral utility profile). Now, given this maximum b-matching, we will match it to a unique maximum flow, $f$ in the modified network. Since each agent $i \in N^{P} \cup N^{O}$ is saturated, send flow $f_{i j}=b_{i}$ on all edges $i j, i \in A^{P O}, j \in B^{P O}$. Now, focus on each component $C_{k}$, if $C_{k}$ has only one node $i$ with utility $U_{i}$. Then this utility is derived only by exchanging with the agents in $B^{O^{\prime}}$. Send a flow, $f_{i j}=x_{i j} \forall i j$ such that $j \in N(i) \cap B^{O^{\prime}}$ where $x_{i j}$ is the number of units exchanged between agents $i$ and $j$ in the given maximum b-matching. On a similar note, we fix the flow for the other components $C_{k}$. We know in any $C_{k},\left|C_{k}\right|-1$ of the vertices are matched in the same component, so a flow of $\left|C_{k}\right|-1$ can be obtained by sending $x_{i j}$ units of flow to the agents in $B^{C_{k}}$. If $i j \in \mu, i \in V^{C}$ and $j \in V^{O}$, then node $i$ is saturated in that particular b-matching; If we send a unit flow in modified network, it is also saturated in the bipartite network. 
To prove the other direction that every integral maximum flow corresponds to a feasible utility profile in the original network. Consider a maximum flow of $|F|$ in the modified bipartite network. First lets say the maximum flow value is $|B|$, this is also the size of the maximum b-matching in the original network. For a given set $A^{C_{i}}$ with $\left|A^{C_{i}}\right|>=2$, there is at most one vertex $a_{j} \in A^{C_{i}}$ such that there exists a vertex $c_{h}^{\prime} \in B^{O}$ with $f\left(a_{j}, c_{h}^{\prime}\right)=1$.In this case, we include in the matching $\mu$. In any maximum flow, for each $C_{i}$, agents in $A^{C_{i}}$ send $\left|C_{i}\right|-1$ units of flow to $B^{C_{i}}$. Consequently from GED lemma, $\left|C_{i}\right|-1$ vertices can be matched among themselves. There are exactly $\left|V^{O}\right|$ units exchanged by agents in $A^{C}$ with agents in $V^{O}$. each such exchnge constitutes one unit flow to $B^{O}$. So all vertices in $V^{O}$ are matched. From GED Lemma, we can match all vertices in $V^{P}$ among themselves in $\mu$. It is easy to see that $u$ is exactly the utility profile corresponding to $\mu$.

Theorem 1 Once we have this, then we can find the lottery mechanism by solving an LP - linear combination of extreme points

Proof: From the lemma above, we have showed that every maximum flow in the modified network is equivalent to a maximum b-matching induced utility profile in the original network. The egalitarian allocation outputs a maximum flow allocation (not necessarily integral). But it is folklore that this non-integral maximum flow can be obtained as a convex combination of integral maximum flows. These convex combinations $\lambda$ are the probabilities with which we pick different matchings from the set of all maximum b-matchings.

\section{$\underline{\text { Lorenz Dominance \& No Envy }}$}

As discussed in Klaus [17], No envy and ETE are not equivalent here. From theorem above, the egalitarian mechanism applied to this bipartite network is a feasible utility profile. Bochet et al. 8] have established the egalitarian mechanism is lorenz dominant among all feasible flows. No Envy also follows from the allocation rule of Bochet et al. [7, 8]

\section{Extensions and Consistency}

Extension of Rules: In the language of Moulin and Sethuraman [27], an allocation rule $\phi$ on a bipartite network $(G, V, E)$ is said to be an extension of the Sprumont's Uniform rule if $\phi$ coincides with the allocation of uniform rule if $G$ is a network with unit demander (supplier) connected to multiple suppliers (demanders). i.e.

$$
\phi_{i}=U_{i}, \quad \forall i \in V
$$

where $U_{i}$ is the utility of agent $i$ under uniform rule.

The notion of "extending a rule" is such that we are not compromising on properties of existing fair allocation mechanisms. Instead, we are actually generalizing in a suitable way to more general network structures. 
In this spirit, Bochet et al. [7, 8, Chandramouli and Sethuraman [12, 11] develop mechanisms which are extensions of the uniform rule. Moulin and Sethuraman [27] study a more general class of extensions of some basic well known rules. We extend this definiton further to general non-bipartite networks. An allocation rule $x$ on a general network $(G, V, E)$ is said to be an extension of the BIMS Egalitarian rule $\phi$ if $x$ coincides with $\phi$ if the network $G$ is bipartite i.e. $x_{i}=\phi_{i}, \quad \forall i \in V$

Lemma 5 The egalitarian rule described earlier for non-bipartite networks is a extension of the probabilitic egalitarian rule (described in appendix) for bipartite networks

Proof: If the network is bipartite, the odd components set, $V^{U}$ forms an independent set. So, $V^{U}$ is a collection of nodes (with peaks $b_{i}$ ) which are not saturated in atleast one maximum b-matching. Hence, the set $B^{U}$ is empty and each agent $i \in V^{U}$ is only connected to agents in $V^{O}$ who are completely saturated. In the bipartite context, if $i$ were a supplier then, any agent $j \in N(i)$ has $x_{j}=b_{j}$ in all pareto allocations. For a detailed derivation of GED for bipartite networks, follow the discussion in Chandramouli and Sethuraman [12]. It is clear from their proof that if the given network $G$ is bipartite, then the Step 1 of our algorithm which modifies the given network into a suitable bipartite network, outputs $G$. Since, we apply the probabilistic egalitarian rule in step 2 , the result follows.

Consistency: As discussed in Chandramouli and Sethuraman [11, the egalitarian rule is an extension of the uniform rule which is not consistent. They propose the edge fair rule which is a consistent extension of the uniform rule to bipartite networks. In the previous section, if after the transformation into bipartite network, if we apply the edge fair mechanism to that network, we would have a consistent extension of the uniform rule to non-bipartite indivisible goods network.

\section{$\underline{\text { Strategic Issues }}$}

\section{Peak Strategyproofness}

In the discussions so far, we have ignored the possibility of agents having control over the values $b_{i}$. Suppose agents report $b_{i}$ to the mechanism designer who then decides the final allocation, then the agents can misreport the peaks to improve their allocation. So far, the allocation of the agents was strictly less than $b_{i}$. If the agents report $b_{i}^{\prime}>b_{i}$, then there is a possibility of agents having an allocation more than his true $b_{i}$. In that case, we need assumptions on the preference profile of agents to compare his utilities when his allocations are on either side of his true peak $b_{i}$.

Single peaked preferences: In the spirit of Bochet et al. [7, 8, we would like to continue our assumption of single peaked preferences for the agent allocations. Mathematically, given a 
prefernce profile $R_{i}$ for agent $i$ and two possible allocations $x_{i}, x_{i}^{\prime}$ then:

$$
\begin{aligned}
& x_{i}^{\prime}<x_{i} \leq p\left[R_{i}\right] \Longrightarrow x_{i} P_{i} x_{i}^{\prime} \\
& p\left[R_{i}\right] \leq x_{i}<x_{i}^{\prime} \Longrightarrow x_{i} P_{i} x_{i}^{\prime}
\end{aligned}
$$

A mechanism is peak strategyproof if it is a dominant strategy for the agents to reveal their peaks truthfully. Given this preference structure, the following example shows that there is no peak strategyproof mechanisms in the set of pareto optimal allocations. Consider agents $(a, b, c)$ connected to each other and peak $b_{i}=1, i \in(a, b, c)$. If the agents report their true peaks, then any allocation mechanism is such that $\left\{\left(x_{a}, x_{b}, x_{c}\right) \mid x_{a}+x_{b}+x_{c} \leq 2\right\}$. W.l.o.g, lets say $x_{a}<1$. Suppose agent $a$ misreports his peak $b_{a}=2$, then we have a unique maximum b-matching and the allocation is $(2,1,1)$. If $2 P_{a} x_{a}$ for agent $a$, he improves his allocation.

\section{$\underline{\text { Link Strategyproofness }}$}

A mechanism is link strategyproof if it is dominant strategy for the agents to reveal all his/her neighbors. Roth et al. [29] established the link strategyproofness of the egalitarian mechanism in the kidney exchange problem. The egalitarian mechanism is not link group strategyproof even in the kidney exchange problem. To see that, consider the following network where each node has 1 unit of good to exchange. Agent $s_{4}$ is matched in every maximum matching, but $s_{7}$ is missed in some maximum matching and recieves a utility strictly less than 1 in the egalitarian allocation. Now, $s_{4}, s_{7}$ can coordinate and misreport about the existence of the link between $s_{4}$ and $s_{3}$. If that link is not reported, then, $\left(s_{4}, s_{5}, s_{6}, s_{7}\right)$ form a separate group and all the agents are matched and receive peak utility. Hence, agent $s_{7}$ improves his allocation.

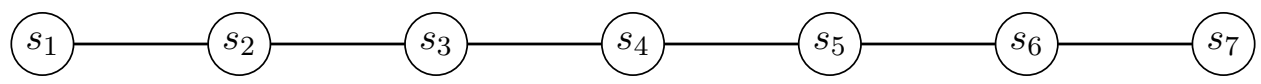

Weak link groupstrategyproof: A mechanism is weakly link group strategyproof if in a coalitional group, every agent who misreports about his connectivity strictly improves his/her allocation.

The agents in over demanded and perfectly demanded component doesn't misreport since they already recieve their peak allocation. The only deviating subsets are those agents in the odd components. Hence, for the rest of the proof we restrict our attention to coalition groups which consists only of agents of the odd components.

Theorem 2 The egalitarian mechanism is weakly link groupstrategyproof

Proof. We prove the result for an arbitrary coalition of agents. Let $A_{i}$ be the set of agents that agent $i$ is linked to, and let $A_{i}^{\prime}$ be agent $i$ 's report. We may assume without loss of generality that any given agent $\in V^{P O}$ finds all the agents in odd components acceptable: if even component 
agent $j$ finds add component agent $i$ unacceptable, then agent $i$ cannot have a link to demander $j$ regardless of his report, so clearly $i$ 's manipulation opportunities are more restricted. Let $\phi$ and $\phi^{\prime}$ be (any) egalitarian flows when the suppliers report $A$ and $A^{\prime}$ respectively, and let $x$ and $x^{\prime}$ be the corresponding allocation to the agents. We show that no coalition of odd component agents can weakly benefit by misreporting their links unless each agent in the coalition gets exactly their egalitarian allocation.

Consider the transformed bipartite network of the given network when going through the analysis below:

The proof is by induction on the number of type 2 breakpoints in the algorithm to compute the egalitarian allocation in the transformed network. Suppose the given instance has $n$ type 2 breakpoints, and suppose $X_{1}, X_{2}, \ldots, X_{n}$ are the corresponding bottleneck sets of suppliers. If $n=0$, every odd component agent is at his peak value in the egalitarian allocation, and clearly this allocation cannot be improved. Suppose $n \geq 1$. Define

$$
\tilde{X}_{\ell}=\left\{i \in X_{\ell} \mid \sum_{j \in A_{i}} \phi_{i j}^{\prime} \geq \sum_{j \in A_{i}} \phi_{i j}\right\}
$$

and

$$
\hat{X}_{\ell}=\left\{i \in X_{\ell} \mid \sum_{j \in A_{i}} \phi_{i j}^{\prime} \leq \sum_{j \in A_{i}} \phi_{i j}\right\}
$$

We shall show, by induction on $\ell$, that for each $\ell=1,2, \ldots, n$ :

(a) $\phi_{i j}^{\prime}=0$ for any $i \in \tilde{X}_{\ell^{\prime}}, j \in \cup_{i^{\prime} \in X_{\ell}} A_{i^{\prime}}, \ell^{\prime}>\ell$; and

(b) $X_{\ell} \subseteq \hat{X}_{\ell}$.

The theorem follows from part (b) above.

Any supplier $k \in X_{\ell} \backslash \tilde{X}_{\ell}$ must have $A_{k}=A_{k}^{\prime}$ as otherwise supplier $k$ is part of the deviating coalition and does worse. Consider now a supplier $i \in \tilde{X}_{\ell}$ with $x_{i}<s_{i}$ and a supplier $k \in X_{\ell} \backslash \tilde{X}_{\ell}$. We have the following chain of inequalities:

$$
\sum_{j \in A_{k}^{\prime}} \phi_{k j}^{\prime}=\sum_{j \in A_{k}} \phi_{k j}^{\prime}<\sum_{j \in A_{k}} \phi_{k j}=x_{k} \leq x_{i}=\sum_{j \in A_{i}} \phi_{i j} \leq \sum_{j \in A_{i}} \phi_{i j}^{\prime} \leq \sum_{j \in A_{i}^{\prime}} \phi_{i j}^{\prime} .
$$

To see why, note that as $k \in X_{\ell} \backslash \tilde{X}_{\ell}$, the second inequality is true by definition, and also $A_{k}=A_{k}^{\prime}$ (justifying the first equality). Also $k, i \in X_{\ell}$ and $x_{i}<s_{i}$, implies $x_{k}<s_{i}$, as suppliers $k$ and $i$ both belong to the same bottleneck set and supplier $i$ is below his peak; this justifies the third inequality. The fourth and fifth inequalities follow from the fact that $i \in \tilde{X}_{\ell}$ and the fact that $\phi_{i j}^{\prime}$ must be zero for all $j \in A_{i} \backslash A_{i}^{\prime}$. This chain of inequalities implies that $x_{k}^{\prime}<x_{k} \leq s_{k}$ and $x_{k}^{\prime}<x_{i}^{\prime}$. Therefore, when the suppliers report $A^{\prime}$, supplier $k$ must be a member of an "earlier" bottleneck set than supplier $i$. An immediate consequence is that demanders in $A_{k}^{\prime}=A_{k}$ do not receive any flow from supplier $i$ when the report is $A^{\prime}$. 
By the induction hypothesis, supplier $i \in X_{\ell}$ does not send any flow to the demanders in $\cup_{1 \leq i^{\prime} \leq \ell-1} \cup_{k \in X_{i}} A_{k^{\prime}}$. Therefore

$$
\left\{j \mid \phi_{i j}^{\prime}>0, j \in A_{i}\right\} \subseteq\left\{j \mid \phi_{i j}>0, j \in A_{i}\right\}
$$

This observation, along with the fact that every $i \in \tilde{X}_{\ell}$ weakly improves, and the fact that $X_{\ell}$ is a type 2 breakpoint implies that $\sum_{j \in A_{i}} \phi_{i j}^{\prime}=\sum_{j \in A_{i}} \phi_{i j}$, establishing (b). Furthermore, in such a solution, every demander $j \in A_{i}$ for $i \in \tilde{X}_{\ell}$ must receive all his flow from the suppliers in $\tilde{X}_{\ell}$.In particular, the demanders in $X_{\ell}$ cannot receive any flow from suppliers in $X_{\ell^{\prime}}$ for $\ell^{\prime}>\ell$, establishing (a). To complete the proof we need to establish the basis for the induction proof, i.e., the case of $\ell=1$. This, however, follows easily: it is easy to verify that the set $X_{1} \backslash \tilde{X}_{1}$ must be empty, so $X_{1}=\tilde{X}_{1}$. As $X_{1}$ is a type 2 bottleneck set, it is not possible for every member of $X_{1}$ to do weakly better unless the allocation remains unchanged. Thus, both (a) and (b) follow.

\subsection{Arbitrary Capacity}

\section{Conclusion}




\section{$5 \quad$ Appendix}

\subsection{Indivisible exchange on bipartite networks: Probabilistic Egalitarian Rule}

We fix a problem $(G, s, d)$ such that $s_{i}, d_{j}>0$ for all $i, j$ (clearly if $s_{i}=0$ or $d_{j}=0$ we can ignore supplier $i$ or demander $j$ altogether). We define independently our solution for the suppliers and for the demanders.

The definition for suppliers is by induction on the number of agents $|S|+|D|$. Consider the parameterized capacity graph $\Gamma(\lambda), \lambda \geq 0$ : the only difference between this graph and $\Gamma(G, s, d)$ is that the capacity of the edge $\sigma i, i \in S_{-}$is $\min \left\{\lambda, s_{i}\right\}$, which we denote $\lambda \wedge s_{i}$. (In particular, the edge from $j$ to $\tau$ still has capacity $d_{j}$ ). We set $\alpha(\lambda)$ to be the maximal flow in $\Gamma(\lambda)$. Clearly $\alpha$ is a piecewise linear, weakly increasing, strictly increasing at 0 , and concave function of $\lambda$, reaching its maximum when the total $\sigma-\tau$ flow is $d_{D_{+}}$. Moreover, each breakpoint is one of the $s_{i}$ (type 1), and/or is associated with a subset of suppliers $X$ such that

$$
\sum_{i \in X} \lambda \wedge s_{i}=\sum_{j \in f(X)} d_{j}
$$

Then we say it is of type 2. In the former case the associated supplier reaches his peak and so cannot send any more flow. In the latter case the group of suppliers in $X$ is a bottleneck, in the sense that they are sending enough flow to satisfy the collective demand of the demanders in $f(X)$ and these are the only demanders they are connected to; any further increase in flow from any supplier in $X$ would cause some demander in $f(X)$ to accept more than his peak demand.

If the given problem does not have any type-2 breakpoint, then the egalitarian solution obtains by setting each supplier's allocation to his peak value. Otherwise, let $\lambda^{*}$ be the first type-2 breakpoint of the max-flow function; by the max-flow min-cut theorem, for every subset $X$ satisfying (19) at $\lambda^{*}$ the cut $C^{1}=\{\sigma\} \cup X \cup f(X)$ is a minimal cut in $\Gamma\left(\lambda^{*}\right)$ providing a certificate of optimality for the maximum-flow in $\Gamma\left(\lambda^{*}\right)$. If there are several such cuts, we pick the one with the largest $X^{*}$ (its existence is guaranteed by the usual supermodularity argument). The egalitarian solution obtains by setting

$$
x_{i}=\min \left\{\lambda^{*}, s_{i}\right\}, \text { for } i \in X^{*}, y_{j}=d_{j}, \text { for } j \in f\left(X^{*}\right),
$$

and assigning to other agents their egalitarian share in the reduced problem $\left(G\left(S \backslash X^{*}, D \backslash f\left(X^{*}\right)\right), s, d\right)$.

That is, we construct $\Gamma^{S \backslash X^{*}, D \backslash f\left(X^{*}\right)}(\lambda)$ for $\lambda \geq 0$ by changing in $\Gamma\left(G\left(S \backslash X^{*}, D \backslash f\left(X^{*}\right)\right), s, d\right)$ the capacity of the edge $\sigma i$ to $\lambda \wedge s_{i}$, and look for the first type-2 breakpoint $\lambda^{* *}$ of the corresponding max-flow function. An important fact is that $\lambda^{* *}>\lambda^{*}$. Indeed there exists a subset $X^{* *}$ of $S \backslash X^{*}$ such that

$$
\sum_{i \in X^{* *}} \lambda^{* *} \wedge s_{i}=\sum_{j \in f\left(X^{* *}\right) \backslash f\left(X^{*}\right)} d_{j}
$$

If $\lambda^{* *} \leq \lambda^{*}$ we can combine this with equation (19) at $X^{*}$ as follows

$$
\sum_{i \in X^{*} \cup X^{* *}} \lambda^{*} \wedge s_{i} \geq \sum_{i \in X^{*}} \lambda^{*} \wedge s_{i}+\sum_{i \in X^{* *}} \lambda^{* *} \wedge s_{i}=\sum_{j \in f\left(X^{*} \cup X^{* *}\right)} d_{j}
$$


contradicting our choice of $X^{*}$ as the largest subset of $S_{-}$satisfying (19) at $\lambda^{*}$.

Once the $\lambda$ is obtained the probabilistic egalitarian rule is obtained similarly to the probabilistic uniform rule of Klaus et al. and Moulin []. The trick here is to randomize at each bottleneck. Now, without loss of generality, let $\bar{X}=\left\{i \in X^{* *} \mid p\left(R_{i}\right) \geq x_{\lambda}+1\right\}=\{1,2 \ldots ., \bar{n}\}$ and $\tilde{N}=\left\{i \in N \mid p\left(R_{i}\right) \leq x_{\lambda}\right\}=\{\bar{n}+1, \ldots, n\}$. Then in the final allocation we obtain, each agent in $\tilde{N}$ receives his peak amount and each agent in $\bar{N}$ receives either $x_{\lambda}$ or $x_{\lambda}+1$. Note that for each $i \in \bar{N},\left(x_{\lambda}+1\right) P_{i} x_{\lambda}$ and that exactly $\bar{n}\left(\lambda-x_{\lambda}\right)$ agents in $\bar{N}$ can receive $x_{\lambda}+1$. The randomized "lottery" places equal probability on all allocations where all agents in $\tilde{N}$ receive their peak amounts, $\bar{n}\left(\lambda-x_{\lambda}\right)$ agents in $\bar{N}$ receive $x_{\lambda}+1$ and the remaining agents in $\bar{N}$ receive $x_{\lambda}$. Hence, the utility profile is obtained by placing equal probabilities on exactly () allocations.

If $p\left(R_{i} \leq x_{\lambda}\right.$, then $U_{i}(R)\left(p\left(R_{i}\right)\right)=1$ and if $p\left(R_{i}\right) \geq x_{\lambda}+1$, then $U_{i}(R)\left(x_{\lambda}+1\right)=\lambda-x_{\lambda}$ and $U_{i}(R)\left(x_{\lambda}\right)=1-\left(\lambda-x_{\lambda}\right)$ The solution thus obtained recursively is the egalitarian allocation for the suppliers. A similar construction works for demanders: We consider the parameterized capacity graph $\Delta(\mu), \mu \geq 0$, with the capacity of the edge $\tau j, j \in D$ set to $\mu \wedge d_{j}$. We look for the first type-2 breakpoint $\mu^{*}$ of the maximal flow $\beta(\mu)$ of $\Delta(\mu)$, and for the largest subset of demanders $Y$ such that

$$
\sum_{j \in Y} \mu \wedge d_{j}=\sum_{i \in g(Y)} s_{i}
$$

etc.. Combining these two egalitarian allocations yields the egalitarian allocation $\left(x^{e}, y^{e}\right) \in \mathbb{R}_{+}^{S \cup D}$ for the overall problem. 


\section{References}

[1] Egalitarian pairwise kidney exchange: Fast algorithms via linear programming and parametric flow.

[2] Atila Abdulkadiroğlu and Tayfun Sönmez. School choice: A mechanism design approach. American economic review, pages 729-747, 2003.

[3] R.K. Ahuja, T.L. Magnanti, and J.B. Orlin. Network flows: theory, algorithms, and applications. 1993.

[4] Mourad Baïou and Michel Balinski. Erratum: The stable allocation (or ordinal transportation) problem. Mathematics of Operations Research, 27(4):662-680, 2002.

[5] Salvador Barberà, Dolors Berga, and Bernardo Moreno. Individual versus group strategyproofness: when do they coincide? Journal of Economic Theory, 145(5):1648-1674, 2010.

[6] Salvador Barbera and Matthew O Jackson. Strategy-proof exchange. Econometrica: Journal of the Econometric Society, pages 51-87, 1995.

[7] O. Bochet, H.Moulin, and R. Ilkılıç. Egalitarianism under earmark constraints,. Technical report, mimeo, 2010.

[8] O. Bochet, H. Moulin, R. Ilkilic, and J. Sethuraman. Clearing supply and demand under bilateral constraints. Technical report, 2010.

[9] A. Bogomolnaia and H. Moulin. Random matching under dichotomous preferences. Econometrica, 72(1):257-279, 2004.

[10] J. Randall Brown. The sharing problem. Oper. Res., 27(2):324-340, 1979.

[11] Shyam Chandramouli and Jay Sethuraman. Strategyproof and consistent rules for bipartite flow problems. http://arxiv.org/abs/1304.3946, 2013.

[12] Shyam Chandramouli and Jay Sethuraman. Groupstrategyproofness of the egalitarian mechanism for constrained rationing problems. Mathematical Social Sciences, 90:111-118, 2017.

[13] S. Ching. An alternative characterization of the uniform rule. Social Choice and Welfare, 11(2):131-136, 1994.

[14] Y. Chun. One-sided population monotonicity, separability, and the uniform rule. Economics Letters, 78(3):343-349, 2003.

[15] William J Cook, William H Cunningham, William R Pulleyblank, and Alexander Schrijver. Combinatorial optimization, volume 33. Wiley. com, 2011. 
[16] B. Dutta and D. Ray. A concept of egalitarianism under participation constraints. Econometrica: Journal of the Econometric Society, pages 615-635, 1989.

[17] L. Ehlers and B. Klaus. Probabilistic assignments of identical indivisible objects and uniform probabilistic rules. Review of Economic Design, 8(3):249-268, 2003.

[18] David Gale and Lloyd S Shapley. College admissions and the stability of marriage. The American Mathematical Monthly, 69(1):9-15, 1962.

[19] Sasaki H. Randomized uniform allocation mechanism and single peaked preferences. Waseda University, Mimeo.

[20] John William Hatfield. Pairwise kidney exchange: Comment. Journal of Economic Theory, 125(2):189-193, 2005.

[21] E. Kalai and E. Zemel. Totally balanced games and games of flow. Mathematics of Operations Research, 7(3):476-478, 1982.

[22] B. Klaus et al. A note on the separability principle in economies with single-peaked. 2006.

[23] Bettina Klaus, Hans Peters, and Ton Storcken. Strategy-proof division with single-peaked preferences and individual endowments. Social Choice and Welfare, 15(2):297-311, 1998.

[24] László Lovász and Michael D. Plummer. Matching theory. AMS Chelsea Publishing, Providence, RI, 2009. Corrected reprint of the 1986 original [MR0859549].

[25] N. Megiddo. Optimal flows in networks with multiple sources and sinks. Mathematical Programming, 7(1):97-107, 1974.

[26] N. Megiddo. A good algorithm for lexicographically optimal flows in multi-terminal networks. American Mathematical Society, 83(3), 1977.

[27] H. Moulin and J. Sethuraman. The bipartite rationing problem. Submitted for publication, 2011.

[28] Herve Moulin and Jay Sethuraman. Loss calibrated rationing methods. 2013.

[29] Alvin E Roth, Tayfun Sönmez, and M Utku Ünver. Pairwise kidney exchange. Journal of Economic Theory, 125(2):151-188, 2005.

[30] T. Sakai and T. Wakayama. A population monotonicity characterization of the uniform rule. 2012.

[31] L. Shapley and H. Scarf. On cores and indivisibility. Journal of mathematical economics, 1(1):23-37, 1974. 
[32] L.S. Shapley and M. Shubik. The assignment game i: The core. International Journal of Game Theory, 1(1):111-130, 1971.

[33] T. Sönmez. Consistency, monotonicity, and the uniform rule. Economics Letters, 46(3):229$235,1994$.

[34] Tayfun Sönmez and Tobias B Switzer. Matching with (branch-of-choice) contracts at the united states military academy. Econometrica, 81(2):451-488, 2013.

[35] Y. Sprumont. The division problem with single-peaked preferences: a characterization of the uniform allocation rule. Econometrica, 59(2):509-519, 1991.

[36] Karol Szwagrzak. Efficient, fair and group strategy proof (re) allocation under network constraints. mimeo.

[37] Karol Szwagrzak. The replacement principle in networked economies under single peaked preferences. mimeo.

[38] Karol Szwagrzak. Strategy-proof allocation under network constraints. mimeo.

[39] William Thomson. Axiomatic and game-theoretic analysis of bankruptcy and taxation problems: a survey. Mathematical Social Sciences, 45(3):249-297, 2003.

[40] Özgür Yılmaz. Kidney exchange: An egalitarian mechanism. Journal of Economic Theory, 146(2):592-618, 2011. 